\title{
Points to consider for prioritizing clinical genetic testing services: a European consensus process oriented at accountability for reasonableness
}

\author{
Franziska Severin ${ }^{1}$, Pascal Borry ${ }^{2}$, Martina C Cornel $^{3}$, Norman Daniels ${ }^{4}$, Florence Fellmann ${ }^{5}$, \\ Shirley Victoria Hodgson ${ }^{6}$, Heidi C Howard ${ }^{7,8}$, Jürgen John ${ }^{1}$, Helena Kääriäinen ${ }^{9}$, Hülya Kayserili ${ }^{10}$, \\ Alastair Kent ${ }^{11}$, Florian Koerber ${ }^{1}$, Ulf Kristoffersson ${ }^{12}$, Mark Kroese ${ }^{13}$, Celine Lewis ${ }^{14}$, Georg Marckmann ${ }^{15}$, \\ Peter Meyer ${ }^{16,17}$, Arne Pfeufer ${ }^{18}$, Jörg Schmidtke ${ }^{19}$, Heather Skirton ${ }^{20}$, Lisbeth Tranebjærg ${ }^{21,22}$ \\ and Wolf $\mathrm{H}$ Rogowski ${ }^{\star, 1,23}$ for the EuroGentest and ESHG/PPPC Priority Consortium
}

Given the cost constraints of the European health-care systems, criteria are needed to decide which genetic services to fund from the public budgets, if not all can be covered. To ensure that high-priority services are available equitably within and across the European countries, a shared set of prioritization criteria would be desirable. A decision process following the accountability for reasonableness framework was undertaken, including a multidisciplinary EuroGentest/PPPC-ESHG workshop to develop shared prioritization criteria. Resources are currently too limited to fund all the beneficial genetic testing services available in the next decade. Ethically and economically reflected prioritization criteria are needed. Prioritization should be based on considerations of medical benefit, health need and costs. Medical benefit includes evidence of benefit in terms of clinical benefit, benefit of information for important life decisions, benefit for other people apart from the person tested and the patientspecific likelihood of being affected by the condition tested for. It may be subject to a finite time window. Health need includes the severity of the condition tested for and its progression at the time of testing. Further discussion and better evidence is needed before clearly defined recommendations can be made or a prioritization algorithm proposed. To our knowledge, this is the first time a clinical society has initiated a decision process about health-care prioritization on a European level, following the principles of accountability for reasonableness. We provide points to consider to stimulate this debate across the EU and to serve as a reference for improving patient management.

European Journal of Human Genetics (2015) 23, 729-735; doi:10.1038/ejhg.2014.190; published online 24 September 2014

\section{INTRODUCTION}

Genetic testing provides multiple benefits to patients and relatives, both from a clinical and a non-clinical perspective. ${ }^{1,2}$ The rapidly expanding availability and application of genetic testing services ${ }^{3-5}$ indicates that these benefits are increasingly made accessible to patients throughout Europe. However, financial resources within publicly funded health-care systems are restricted. Although technical improvements are leading to a decrease in laboratory costs per test, the evidence that genetic tests produce overall savings in health-care systems is currently weak ${ }^{6}$ and limited to a minority of tests. ${ }^{7,8}$ Rather, the costs of data analysis and storage, interpreting the test results, counseling services and follow-up care could lead to an increase in the overall health-care costs associated with genetic testing. ${ }^{9,10}$ Therefore decisions have to be made about which tests should be covered by the public health-care budgets.

To date there is a lack of structured guidance for the decisionmakers on how to give priority to genetic tests within clinical genetic services. In a Canadian survey, health-care providers reported that given the absence of coordinated approaches, resource allocation decisions are often left to local providers of genetic tests. ${ }^{11}$ It is likely

${ }^{1}$ Institute for Health Economics and Health Care Management, Helmholtz Zentrum München, German Research Center for Environmental Health (GmbH), Neuherberg, Germany; ${ }^{2}$ Department of Public Health and Primary Care, KU Leuven, Leuven, Belgium; ${ }^{3}$ Department of Clinical Genetics, Section Community Genetics, EMGO Institute for Health and Care Research, VU University Medical Center Amsterdam, Amsterdam, The Netherlands; ${ }^{4}$ Department of Global Health and Population, Harvard School of Public Health, Boston, MA, USA; ${ }^{5}$ Service de Génétique Médicale, CHUV, Lausanne, Switzerland; ' 6 Department of Medical Genetics, St George's University of London, London, UK; ${ }^{7}$ Center for Research Ethics and Bioethics, Uppsala University, Uppsala, Sweden; ${ }^{8}$ Université de Toulouse 3 Paul Sabatier, UMR 1027, Faculté de médecine Purpan, Toulouse, France; ${ }^{9}$ National Institute for Health and Welfare, Helsinki, Finland; ${ }^{10}$ Department of Medical Genetics, Istanbul Medical Faculty, Istanbul University, Istanbul, Turkey; ${ }^{11}$ Genetic Alliance UK, Unit 4D, Leroy House, London, UK; ${ }^{12}$ Department of Clinical Genetics, Laboratory Medicine, Region Skane and Lund University, Lund, Sweden; ${ }^{13}$ PHG Foundation, 2 Worts Causeway, Cambridge, UK; ${ }^{14}$ Clinical and Molecular Genetics, UCL Institute of Child Health and Great Ormond Street Hospital for Children NHS Foundation Trust, London, UK; ${ }^{15}$ Institute for Ethics, History and Theory of Medicine, Ludwig Maximilians University Munich, Munich, Germany; ${ }^{16}$ Human Genetics Foundation, Munich, Germany; ${ }^{17}$ Institute for Medical Genetics, University Medicine, Rostock, Germany; ${ }^{18}$ Institute for Bioinformatics and Systems Biology, Helmholtz Zentrum München, German Research Center for Environmental Health $(\mathrm{GmbH})$, Neuherberg, Germany; ${ }^{19}$ Department of Human Genetics, Hannover Medical School, Hannover, Germany; ${ }^{20}$ Faculty of Health and Human Science, School of Nursing and Midwifery, Plymouth University, Drake Circus Plymouth, UK; ${ }^{21}$ Department of Audiology, Bispebjerg Hospital/Rigshospitalet, Copenhagen, Denmark; ${ }^{22}$ Institute of Cellular and Molecular Medicine, University of Copenhagen, Copenhagen, Denmark; ${ }^{23}$ Institute and Outpatient Clinic for Occupational, Social and Environmental Medicine, Clinical Center, Ludwig Maximilians University, Munich, Germany

*Correspondence: Dr WH Rogowski, Institute for Health Economics and Health Care Management, Helmholtz Zentrum München, German Research Center for Environmental Health (GmbH), Neuherberg, Germany. Tel: +49 893187 4128; Fax: +49 893187 3375; E-mail: rogowski@helmholtz-muenchen.de

Received 9 April 2014; revised 11 August 2014; accepted 19 August 2014; published online 24 September 2014 
that this is also the case in many European health-care systems. ${ }^{12}$ In the face of resource constraints, prioritization decisions are likely to be based on contingencies of daily practice rather than on well-reflected considerations. Also, there is a risk that without a shared set of prioritization criteria, decision makers may be accused of overprovision by health-care financers or of under-provision of genetic services by patients. Given this situation, it has been argued that the development of agreed standards for resource allocation decisions based on sound medical, economic and ethical considerations would be beneficial for both decision-makers and those who potentially benefit from health care. ${ }^{13}$

The question as to how health-care services should be given priority has a long history of debate within the literature. The challenge for prioritizing genetic tests is to determine a set of criteria that can be applied to rank order different tests according to their relative priority. Even if theoretical and empirical work on such criteria is available in the literature, ${ }^{14,15}$ it has been argued that reasonable people may still disagree about which criteria should be applied and, if more than one criterion applies, about the relative weight they should receive. ${ }^{16}$ Therefore, these issues have to be resolved in a fair decision procedure to obtain legitimate guidance. A widely cited framework of procedural fairness is 'accountability for reasonableness' (A4R). ${ }^{17}$ The following paper describes the results of a consensus process that has been organized according to this framework. The aim of the process was to develop a shared set of criteria and considerations for prioritizing genetic tests which can be used on a regional and local level of decision making in the absence of clearly defined national prioritization guidance.

\section{MATERIALS AND METHODS}

According to Daniels and Sabin, ${ }^{16,17}$ a decision process complies with A4R if it meets the following four conditions: relevance, publicity, revision and appeal and enforcement. The following section illustrates how these conditions were addressed during this decision process about a general prioritization framework for genetic services. Briefly, the process was based on five steps (see Figure 1): (1) identifying intuitively plausible and practically relevant prioritization criteria; (2) developing a theoretical framework for reasonable prioritization; (3) establishing evidence about tentative weights these prioritization criteria should have; (4) stakeholder and expert deliberation about the steps (1)-(3); and (5) website publication of the results to allow for revision and appeal. The central part of step (4) was a two-day stakeholder and expert workshop (from 28 to 29 November 2012) with 25 participants which was part of the activities conducted within EuroGentest (www.eurogentest.org).

\section{Relevance}

The relevance condition of A4R requires that the prioritization rests on evidence, reasons and principles that fair-minded people can agree are relevant for patient care under resource constraints. ${ }^{17}$ Hence as a starting point, interviews with clinicians and patient representatives were conducted to elicit criteria which are relevant to the most important stakeholders. To obtain a general overview of the patient perspective, we focused on communicating with patient representatives rather than patients themselves. ${ }^{15}$ Furthermore, an explorative literature review assessed normative frameworks relevant for prioritizing genetic services in a reasonable and fair manner ${ }^{14}$ and a theoretical prioritization framework was developed. The criteria which appeared most relevant were operationalized and weighted within a discrete-choice experiment (DCE) ${ }^{15,18}$ The statistically significant impact of the criteria described in the DCE served as a confirmation of the criteria's relevance. To prepare for the discussion, the criteria were shared with the workshop participants prior to the meeting, alongside with a survey regarding the participants' views and experiences with prioritization practice.

Scientific publication of this document also contributes to the relevance criterion, because it facilitates further debate in a transparent way.

\section{Publicity}

The publicity condition requires that the rationales for resource allocation decisions are accessible to the relevant stakeholders. ${ }^{17}$ It was assumed that health-care providers and patients are the most important stakeholders for the vertical prioritization of budgets for genetic services. Further stakeholders include health-care funders, regulatory agencies, industry representatives and the general population. Information about this prioritization activity toward these stakeholders was provided through different channels such as relevant newsletters, websites (eg the EuroGentest website) and personal communications to the relevant stakeholders. Furthermore, it was presented at the EuroGentest General Assembly March 2013 in Prague and at the Annual Conference of the European Society of Human Genetics (ESHG) June 2013 in Paris.

\section{Revision and appeal}

Those affected by a decision should have the chance to challenge it on the basis of relevant arguments that have not been considered duly during the original decision-making process. ${ }^{17}$ To ensure that as many rationales as possible were considered during the workshop, a broad range of expertise (clinical genetics, molecular genetics, economics, ethics, public health and sociology) and various stakeholders (clinicians, patient representatives, health technology assessment agencies and neutral experts who can contribute views from the informed general population) were involved in the workshop and its preparation (see Supplementary Appendix Table 1 for a list of participants). They also included members of the Public and Professional Policy Committee of the ESHG Unanimous assent was reached, ensuring that the value judgments of all the included stakeholders were accounted for.

Preliminary versions of this recommendation statement were shared for comments and revisions among the workshop participants as well as on the website of the ESHG and EuroGentest. A broad range of stakeholders were contacted by email and invited to comment. The document was then submitted to the standard process of ESHG recommendations including approval by the ESHG board. The preliminary version and all written stakeholder comments made before the final version are available from the first author upon request.

\section{Enforcement}

Apart from the conditions of publicity, relevance and revision and appeals, A4R requires that appropriate regulation is in place to ensure that the prioritization decisions comply with these conditions. To facilitate enforcement for this rather singular process of developing a general decision framework for prioritization decisions, this study draws upon the standard process for documents to formally become ESHG statements. These processes always have to include website publication (publicity), open discussion on the website (relevance) and incorporation of comments (revisability/appeals). The following sections outline the results of the consensus process on prioritization criteria for genetic tests.

\begin{tabular}{|l|l|l|}
\hline Identifying relevant \\
prioritization criteria: \\
Literature review and explorative \\
interviews
\end{tabular}

Figure 1 Overview of prioritization process. 


\section{RESULTS}

\section{Scope and context of prioritizing genetic tests}

In the following, 'genetic tests' refer to genetic services as a whole. This includes, first, the analysis of human DNA, RNA, chromosomes, proteins or certain metabolites to detect heritable disorders or treatment responses (the laboratory testing process). Second, it includes the process of analyzing and interpreting the tests result. Third, it includes informing the patient about the test results and the implications for his or her life, a procedure which may differ among diagnoses and may include genetic counseling. We excluded transgenerational aspects of genetic testing (eg pre-implantation and prenatal testing) as these tests involve a range of very specific issues including concerns about eugenics that are discussed elsewhere. Also, population screening programs were excluded as they are expected to undergo separate kinds of prioritization considerations in comparison with other public health programs.

Prioritization should be preceded by efforts to improve the efficiency of care. This includes a focus on tests with both sufficient test performance and validity, that is, the sensitivity and specificity of the test and scientific validity for the analysis, and on information regarding the clinical implications of the test result. Confidence in the test's performance and the implication of its results is particularly relevant for multifactorial diseases where it is often unclear how the discoveries can be used to improve the patient treatment as the positive and negative predictive values of the test results are small, while the confidence intervals of the prediction are wide. Recently, the ESHG made a recommendation against the use of tests for multifactorial conditions, where evidence of the clinical impact on patient benefit are not sufficiently met. ${ }^{19}$

Prioritization of genetic tests here is understood as placing tests into a rank order or into rank-ordered categories. Prioritization of genetic services (and, consequently, exclusion of others from public funding) can occur in different ways. For example, local decision-making committees of health-care providers may determine whether a testing service is provided or excluded from provision at the expense of the existing budget. Alternatively, prioritization can be the basis to limit the scope of a test (eg testing is only provided for the most frequent pathogenic variants rather than the whole gene). Furthermore, it can used to assign services to different priority categories which determine the place of the testing service on a waiting list, so that individuals who seek testing may wait longer or shorter periods of time depending on the anticipated suffering from avoidable disease and uncertainty. It is important to realize that prioritization is not a one-way road to limiting services: Tests with a high priority should be made available to all individuals who might benefit from it.

This prioritization activity focuses on the efficient and fair allocation of collectively funded resources (eg taxes or contributions to statutory health insurance) on a regional or local level (eg to inform decisions by commissions on the management of genetics services of a hospital's genetics unit which has a fixed budget to spend). As reimbursement rules typically differ across health-care systems, this guideline can only serve as a complementary decision aid and cannot replace existing national coverage and prioritization standards.

These points to be considered are intended for situations where not all desirable genetics services that are technically available can be offered to all patients who may need them because of a lack of funding (eg, because there are too few geneticists to see all patients or because there is a lack of funding to pay the lab test).

\section{Relevant criteria for prioritizing genetic tests}

The workshop participants and commentators on this document agreed that medical benefit, health need and costs (see Table 1) should be taken into consideration for prioritizing genetic services. In general, it was considered desirable to operationalize and weight these criteria using empirical methods, so that genetic tests can be ranked in a scientifically reflected manner. However, a number of points have to be considered before these criteria can be used for prioritizing genetic tests.

\section{Medical benefit}

Patients can derive many benefits from genetic testing. When conducting a benefit assessment, it is important not only to include the positive effects but also to weight benefits against the harms that might be associated with the genetic test. ${ }^{1}$

\section{Clinical benefit for the tested individual}

From a medical perspective, the distinction between a test where an effective clinical treatment or prevention options exist and a test where the disease progression cannot be altered with preventive measures or by treatment is of substantial importance. Detecting an individual with a condition for which medical interventions are available should have higher priority than identifying an individual with a similar risk of a condition with no evidence that the disease can be prevented or that its course can be altered in a favorable way.

In some cases, particularly for rare disorders and for long-term benefits, the evidence for the clinical benefit might be hard to establish. Also, individual behavior patterns need to be accounted for when assessing the benefit of a genetic test. Although there might be a benefit from the genetic test per se, some individuals might not have it because they do not accept the next step of treatment or prevention or just do not want to know. The clinical benefit of genetic testing also includes reduction in anxiety if a close relative of a patient with a severe hereditary disease is diagnosed not to carry the mutated allele, and no longer needs clinical follow-up.

\section{Non-clinical benefit for the tested individual}

Similar to some other kinds of diagnostics, genetic testing can also provide large benefits that are not covered by existing measures of clinical benefit. An integrated part of the clinical care is to provide individuals with a more accurate prognosis concerning life span and quality of life. Although there might not be a treatment or prevention option available, the diagnosis itself may, in some cases, enable the individuals and families to plan their lives in light of what is known about the particular condition. This may be the case with Huntington disease, where the test result provides information that could help to make decisions even if it does not, in itself, alter the natural course of the condition. Such non-clinical consequences are frequently labeled as patient empowerment, ${ }^{20}$ because they enable the patients to get a better mental and emotional control over their health and health care, and account for other aspects of their lives which are influenced by their current and future health status. These consequences should also be considered in appraising the priority of a genetic test. Non-clinical benefits may also arise from information which neither leads to any action of clinicians nor of patients. Also here, further evidence is needed and the priority that tests with such benefits should have needs to be determined.

\section{Benefits for family members}

The benefits from genetic testing may also apply to individuals other than the index patient. Cascade testing of close relatives of an 
Table 1 Points to consider for prioritizing genetic tests

\begin{tabular}{|c|c|c|}
\hline Criterion & Explanation & Selected challenges for measurement and use \\
\hline \multicolumn{3}{|l|}{ Medical benefit } \\
\hline Clinical benefit for tested individual & $\begin{array}{l}\text { Tests where, based on the results, effective clinical treatment or } \\
\text { prevention options exist, should be of higher priority than tests } \\
\text { without effective interventions. }\end{array}$ & $\begin{array}{l}\text { For rare diseases and long-benefit, evidence frequently is weak. } \\
\text { Individual behavior patterns (eg whether the treatment would be } \\
\text { pursued) need to be accounted for to establish the benefit of a } \\
\text { genetic test. }\end{array}$ \\
\hline Non-clinical benefit for tested individual & $\begin{array}{l}\text { Consideration of benefit also should include health-related infor- } \\
\text { mation by itself which may assist individuals and families in } \\
\text { planning their life. }\end{array}$ & $\begin{array}{l}\text { There is a need for more scientific evidence about the benefit for life } \\
\text { decision making ('empowerment') incurred by different genetic tests. } \\
\text { It is unclear how non-actionable information should be accounted for. }\end{array}$ \\
\hline Benefit for family members & $\begin{array}{l}\text { Consideration of benefit also should include benefits for the family } \\
\text { members. }\end{array}$ & $\begin{array}{l}\text { Also, the non-clinical benefits may apply to relatives. } \\
\text { Particularly for tests because of non-medical benefits, trade-offs with } \\
\text { concerns for privacy or the right not to know may be relevant. }\end{array}$ \\
\hline Likelihood & $\begin{array}{l}\text { Tests for patients with high a-priori risk of developing the disease } \\
\text { (eg high-risk populations or even first-degree relatives) should be } \\
\text { of higher priority than tests for patients with low a-priori risk. }\end{array}$ & $\begin{array}{l}\text { Incomplete penetrance, that is, a low probability of overt symptoms } \\
\text { needs to be incorporated in risk assessment. } \\
\text { High-risk individuals may be more likely to be detected as genetic } \\
\text { cases in clinical practice than medium-risk individuals. }\end{array}$ \\
\hline Time window & $\begin{array}{l}\text { It may be that the benefit from a test can only be obtained within } \\
\text { a finite time window. }\end{array}$ & $\begin{array}{l}\text { Criterion likely to be of higher relevance for a local perspective which } \\
\text { also includes management issues than from a health-care system } \\
\text { perspective. }\end{array}$ \\
\hline \multicolumn{3}{|l|}{ Health need } \\
\hline Severity & $\begin{array}{l}\text { Tests for conditions with a severe impact on the patient's health } \\
\text { should be of higher priority than tests for comparatively mild } \\
\text { diseases. }\end{array}$ & $\begin{array}{l}\text { Severity can be expressed in reduced life expectancy or in reduced } \\
\text { health related quality of life. Methodological details of measuring } \\
\text { severity need to be considered because they can cause concerns, for } \\
\text { example, if QALYs are used. }\end{array}$ \\
\hline Progression & $\begin{array}{l}\text { Tests for conditions which may already have developed silently or } \\
\text { even overtly in the tested individual should be of higher priority } \\
\text { than tests in healthy individuals before the onset of disease. }\end{array}$ & $\begin{array}{l}\text { Needs to be applied in context-dependent manner: } \\
\text { At a certain stage of progression clinical signs and symptoms may be } \\
\text { more accurate and make genetic testing obsolete. } \\
\text { May be inversely related to medical benefit if benefit primarily arises } \\
\text { from prevention in healthy or presymptomatic carriers. }\end{array}$ \\
\hline \multicolumn{3}{|l|}{ Costs } \\
\hline Costs & $\begin{array}{l}\text { Tests with lower costs (and, thus, less other tests displaced) } \\
\text { should be of higher priority than high-cost tests. }\end{array}$ & $\begin{array}{l}\text { Consideration of costs should include savings elsewhere in health-care. } \\
\text { Further evidence from well-designed economic evaluations are needed. } \\
\text { Costs should not be the dominant but only one criterion among others. }\end{array}$ \\
\hline Weighting the criteria & \multicolumn{2}{|c|}{$\begin{array}{l}\text { Typically, tests differ across different criteria simultaneously so that the criteria need to be weighted. A score based on empirical methods of } \\
\text { measuring and weighting the criteria would be desirable. However, currently, it is too premature for such quantitative ranking algorithm, for } \\
\text { example, because of too limited evidence about whether the criteria are met and unresolved questions about which value judgments should } \\
\text { be incorporated into the empirical analysis. }\end{array}$} \\
\hline
\end{tabular}

individual with a genetic condition helps to effectively identify additional carriers of pathogenic variants. For example, in the case of Lynch syndrome, testing is conducted not only for the benefit of the index case but also for his or her relatives. This is because carriers can choose to undertake increased colonoscopic surveillance which improves their expected health outcomes. ${ }^{21,22}$

Also, non-clinical benefits may apply to the relatives of an index case if the disease risk of a relative is established. If in a family with suspected Lynch syndrome a pathogenic variant was detected so that relatives can undergo testing, some would experience relief from not carrying the familial variant and removal of the need for regular invasive surveillance. However, particularly in the case of tests because of non-clinical benefits for family members, relevant trade-offs with concerns for privacy or the right not to know may have to be taken into consideration.

\section{Likelihood of disease}

One indicator of the size of benefit which can be obtained by a genetic test is the patient's a-priori risk of being tested positive for the condition. Identifying individuals at increased risk is of key importance as testing high-risk groups improves the performance of the test in terms of the cases detected. Therefore, it seems to be justified to give priority to individuals who are likely to be at substantially greater risk of having a condition than others in the population (in particular, close relatives). Also, high-risk subpopulations can be considered of higher priority for genetic testing than individuals in populations with lower pathogenic variant prevalence.

Particularly for predictive/presymptomatic testing, it should be noted that 'likelihood of disease' refers to the risk of the phenotype. Therefore, incomplete penetrance, that is, a reduced probability of overt symptoms in people who have the underlying genetic defect, directly affects the risk assessment. Testing an individual with an elevated risk of a low-penetrance condition might be of lower priority than testing an individual with an equivalent risk of a pathogenic variant with a full-penetrance condition. Also, it needs to be considered that a familial aggregation in presence of a pathogenic variant might confer a higher individual risk for a relative to develop the condition than in the absence of a positive family history. 
This might, for example, be the case for pathogenic variants in the CHEK2 gene and breast cancer. ${ }^{23}$

Furthermore, it may need to be considered that population groups with high pathogenic variant probabilities are more likely to be recognized as genetic cases in the clinical environment than those with lower probabilities and therefore will not benefit from special patient care. Therefore, also this criterion may need to be adapted to the context.

\section{Time window to obtain benefit}

It may be that the benefit from a test can only be obtained within a finite time window. This is the case if there is a restricted time in which the clinical reaction on a positive diagnosis can still be effective. For example, in a newborn at risk of an inherited error of metabolism who (in the case of a positive diagnosis) would immediately need a special diet there is a restricted time window in which testing can be conducted. Tests may be less urgent if therapy can be started somewhat later without negative effects on the course of the disease. Timing is, therefore, also a relevant aspect for priority setting.

\section{Health need}

Besides the medical benefit, also health need should be considered which can exist independently of whether a treatment is available to improve the patient's health. Two aspects of health need appeared most important for prioritizing genetic tests.

\section{Severity of the disease}

One important aspect is the impact that the condition targeted by the test has on the patient's health. It can be assumed that individuals at risk of a severe condition are worse off than those at risk of a milder condition. Therefore testing for severe conditions should be assigned higher priority than testing for milder conditions. It was generally agreed that the severity of a genetic condition can be expressed in terms of reduced life expectancy, in reduced health related quality of life or a combination of these two. While some diseases could lead to greatly reduced life expectancy, other conditions can confer long-term disabilities. To compare these diseases, 'severity' needs to be measured. However, methodological details of measuring severity can cause concerns; for example, concerns that have been expressed against the use of quality-adjusted life years (QALYs) if this metric was used. Also, it needs to be determined to what extent 'severity' should be based on objective measures or on the subjective patient experience.

\section{Progression of disease}

Another aspect of health need that should be considered is how immediate the potential health loss is at the time of testing. This can be estimated by the extent to which the condition may already have progressed in the tested individual. The more advanced a disease is likely to be, given the patient's age and potential symptoms, the higher the priority of the test.

However, this can be highly context-sensitive; beyond a certain stage of progression, clinical signs and symptoms may be more accurate and make genetic testing redundant. Also, health need in terms of progression may be negatively correlated with health benefit: in the case of hereditary cancer, for example, genetic tests are likely to be most important for healthy individuals because prevention is most effective at early stages of cancer development.

\section{Costs}

Conducting one test in a situation of resource scarcity where not all tests can be funded implies that there are other tests which are omitted and the benefits of these other tests are thus forgone. Therefore, also the resources consumed by a genetic test (and thus unavailable for alternative tests) should be considered.

Ideally, costs should be considered from a societal or at least a health-care system perspective, including all costs that are associated with a genetic testing service (eg counseling, follow-up testing and treatment). Also benefits should ideally be considered at a similar level. From a societal perspective, costs may be much higher than for the genetic test alone, but they may also come with cost savings elsewhere in the health-care system or society, for example, owing to disease prevention. Also, early diagnosis of the condition could result in costs savings because patients would not continue seeing other doctors and undergo other tests that incur costs to the health system. The test result that an individual is not a carrier might also lead to cost savings if otherwise indicated measures and interventions are not implemented.

However, to be confident about the overall additional costs or cost savings, well-designed health economic evaluations are necessary. ${ }^{7,9}$ In practice, information about resource consumption over the whole care process is likely to be limited so that it may be necessary to restrict the assessment of costs to the total costs from a health-care provider perspective, including only the relevant scarce budgets for laboratory testing and counseling.

To explore the opportunity costs of the current service patterns, discussions about prioritization should attempt to incorporate information about the types of resource constraints and the types of tests currently withheld due to these resource constraints. The priority of these tests forgone can serve as one qualitative benchmark for deciding whether a new test should be funded from limited resources.

The overall budget impact of a genetic test is also relevant such that, the higher the budget impact, the better the evidence of the aforementioned relevant criteria should be in order to support its coverage. This is also to avoid discrimination against rare diseases which may have less evidence available but also less budget impact.

\section{Establishing the relative importance of criteria}

Genetic tests typically differ by more than one of these criteria simultaneously. Some testing situations might be clearly dominated by others, for example, in the case of testing a first-degree relative for a severe and treatable condition compared with testing someone without a family history for a mild and untreatable condition. However, most testing situations are likely to be less obvious. The resulting order of priority then depends on the weights given to each of these criteria.

Generally, empirical methods such as DCEs can be used to appraise the relative importance of one criterion over the other. ${ }^{15}$ Results from the preparatory DCE among patients and clinicians indicated that participants attached particularly high value to a proven medical benefit of the test, high risk of having the disease and low costs of the test. ${ }^{15}$ The field of organ transplantation is an example where sophisticated algorithms have been developed and are used for allocating a scarce resource. ${ }^{24}$

At the current point in time however, it is premature to propose such an algorithm for genetic tests. This is, for example, because the evidence regarding the extent to which the criteria above are met is still too weak to allow for a valid quantitative ranking of tests. Also, fair and reasonable prioritization is highly context-specific and may depend on multiple further issues for which it is currently unclear how they can best be incorporated into or addressed alongside the use of such a prioritization algorithm. 


\section{How to use this prioritization framework}

At this point in time, the public discussion about priority setting in Europe is still at an early stage and there is neither consensus about the most appropriate ethical frameworks nor the economic tools and their practical implementation. Moreover, genetic services are currently undergoing tremendous technological change and the decision contexts are highly heterogeneous across European health-care systems. Also, it is hardly possible to predict which further points have to be considered in future. Therefore, it is premature to give clear recommendations about how genetic tests are to be prioritized. Instead, these criteria can be used for three purposes; first, they provide points to consider when prioritization decisions have to be made, for example, at meetings on an annual basis when existing procedures for patient management are reviewed. Not only the criteria per se but also the procedural framework of A4R can provide a valuable orientation of fair and reasonable decisions about prioritizing genetic services. Second, they can serve as a starting point for the further development of quantitative approaches such as ranking lists established on the basis of DCE results. Third, these points to consider can serve as a valuable basis for discussing current priorities for genetic care in order to identify which services are indispensable and where there is room for improvement regarding equitable access to highpriority services. Going through the exercise of matching past decisions against the suggested set of criteria within a team of clinicians or regional administrators of public health funds can also have a positive impact on the consistency of allocation decisions, even if it is unlikely (and not necessarily desirable) that all subjectivity and the need for prudential judgment can be eliminated.

\section{DISCUSSION}

Throughout Europe, clinicians are faced with resource constraints and the problem of implicit prioritization of health-care services. Although whole genome sequencing techniques lead to steadily decreasing laboratory costs, the increasing volume of tests as well as the costs associated with the efforts and expertise to interpret complex test results could lead to an overall increase in health-care expenditure. ${ }^{10}$ Therefore prioritization criteria similar to those discussed above might still be needed if genetic tests based on whole genome sequencing are used on a larger scale in clinical practice. Both, the new types of diagnostics and the increasing availability of new interventions is likely to drive the need for priority setting.

To our knowledge, this is the first time a clinical society has responded actively to this challenge on a European level. The decision process resulted in points to consider for prioritizing genetic tests. As it is currently premature to develop definite and structured recommendations, these points to consider are intended to stimulate a longer process of identifying acceptable prioritization tools. At this point in time, the results from this project are therefore just the first step toward a more harmonized and equitable provision of highpriority genetic services.

\section{Implications for future prioritization activities}

Genetic exceptionalism for prioritization?. This study developed an approach for vertical prioritization of genetics services only. Generic measures that can inform prioritization between a range of medical technologies often rely on generic concepts such as costs per QALYs. Although such generic concepts can also be used to assess the medical benefit of a genetic test, they are of limited sensitivity to the multifaceted benefits and relevant issues in the provision of genetic tests. Also, the applicability of condition-neutral frameworks is limited if budgets are assigned to different clinical areas, such as genetic services, and prioritization has to take place within these budgets. This framework may provide a more acceptable alternative because it accounts for specific attributes of genetic tests.

There are also genetic services which are integrated into other clinical areas such as genetic screening programs which are funded from public health service budgets. Here, it is more likely that their benefits and costs have to be balanced against the benefits and costs of very different alternatives. Therefore, the use of more conditionneutral tools is more likely to be applicable. Nevertheless, maximization of health outcomes like QALYs is unlikely to be considered acceptable by all participating stakeholders. ${ }^{25}$ This study can help to identify relevant points that should also be considered. Criteria of health need, such as the potential progression of disease or benefit in terms of information for life decision making without tangible health benefit is likely to be relevant for other diagnostics as well.

Using accountability for reasonableness for prioritization. To our knowledge, this is also the first time an international organization like the ESHG has specifically applied the widely cited 'accountability for reasonableness' framework ${ }^{17}$ to improve the legitimacy of the recommendations for priority setting.

This normative framework is sufficiently generic to allow for very different ways of implementation. In this context, it appeared very consistent with standards of good scientific practice. This case study may therefore also serve as an example for prioritization processes in other clinical areas.

\section{Implications for further research}

Genetic testing can provide large clinical and non-clinical benefits for the tested individuals and their family members, both in case of a positive and a negative test result. However, it is very unlikely that in near future for all or most genetic tests a statistically significant establishment of this benefit can be achieved. Particularly for benefits that are not strictly clinical (yet still related to health, such as anxiety and so on), the evidence is still weak. Instruments for measuring this type of benefit are needed in a context of scarce resources and evidence-based medicine where technologies without good evidence of benefit may be candidates for exclusion from coverage.

Also, there is little evidence about the total effects and costs of genetic services from a health-care system perspective-a recent review of economic evaluations of genetically targeted interventions has concluded that the use of genetic tests for personalizing medicine does not per se provide high or low value for money. Instead, the costeffectiveness of genetic tests largely depends on how genetic tests are used in the care process. ${ }^{26}$ In the face of falling test costs and improved means of prevention, there may be many applications where whole genome sequencing techniques are not only beneficial for patients but also highly cost-effective or even cost-saving. More health economic evidence is needed to identify these applications and more methodological work is needed to validate projected effects and costs alongside their use in practice.

The lack of evidence for a criterion should not necessarily lead to exclusion of a test from coverage. This is particularly true for very-rare diseases, where a lower threshold of evidence as part of the evaluation process might be acceptable because the number of patients is too small to conduct reliable clinical studies. A future challenge that needs to be resolved is therefore, how the evidence gap can be filled in a reasonable and fair manner.

Furthermore, this study revealed the need for standards regarding the specification and operationalization of the prioritization criteria. For example, 'severity of disease' can be measured according to 
different concepts which could result in different rank orders. ${ }^{27}$ Also, further work is needed to explore the applicability of this framework for prioritizing genetic tests in other medical specialties.

Although A4R is highly cited in the literature, there is still little known about its impact on decisions and its acceptance in health-care practice. First evidence suggests that transparency and participation does indeed increase the reasonableness of decision criteria. ${ }^{28}$ Further research is necessary, for example on the impact of decision outcomes if other stakeholders are included which could be assessed within further workshops. Also, the acceptability of the final results from this guideline as well as its uptake and impact of use in healthcare practice needs to be assessed further.

\section{CONCLUSION}

Explicit, fair and reasonable priority setting of health-care resources in Europe is still at an early stage. The results from this study provide important points to consider for prioritizing genetic tests and highlight issues that need further development.

Within this study we provide results from a decision process oriented at the A4R principles about prioritization criteria for genetic testing services. Key criteria were evidence of clinical benefit for the individual being tested, benefit for planning one's life, benefit for other persons and timing to obtain the benefit, the likelihood of disease or benefit, severity and progression of the disease and the costs of the test. These criteria should not be seen as fixed or final, but rather as a starting point for further discussions toward a more harmonized and considered approach to priority setting for genetic services across the EU.

\section{CONFLICT OF INTEREST}

The authors declare no conflict of interest.

\section{ACKNOWLEDGEMENTS}

We acknowledge the stakeholders who participated in the workshops and are grateful for the insights they provided. This study was supported by EuroGentest (EuroGentest 2, Unit 2: Genetic testing as part of health care, Work package 6 (WH Rogowski), grant number Health-F4-2010-261469). It was further supported by the grant 'Individualized Health Care: Ethical, Economic and Legal Implications for the German Health Care System' of the German Federal Ministry of Education and Research (BMBF, grant numbers 01GP1006A and B).

1 Rogowski WH, Grosse SD, John J et al: Points to consider in assessing and appraising predictive genetic tests. J Community Genet 2010; 1: 185-194.

2 Grosse SD, Wordsworth S, Payne K: Economic methods for valuing the outcomes of genetic testing: beyond cost-effectiveness analysis. Genet Med 2008; 10: 648-654.

3 Schmidtke J, Pabst B, Nippert I: DNA-based genetic testing is rising steeply in a national health care system with open access to services: a survey of genetic test use in Germany, 1996-2002. Genet Test 2005; 9: 80-84.

4 Javaher $\mathrm{P}$, Kaariainen $\mathrm{H}$, Kristoffersson $\mathrm{U}$ et al: EuroGentest: DNA-based testing for heritable disorders in Europe. Community Genet 2008; 11: 75-120.

5 Spending on genetic tests grows: report calls for more genetics education and counselors. Am J Med Genet A 2012; 158A: ix.
6 Assasi N, Schwartz L, Tarride JE, Goeree R, Xie F: Economic evaluations conducted for assessment of genetic testing technologies: a systematic review. Genet Test Mol Bioma 2012; 16: 1322-1335.

7 Rogowski W: Genetic screening by DNA technology. A systematic review of health economic evidence. Int J Technol Assess Health Care 2006; 22: 327-337.

8 Carlson JJ, Henrikson NB, Veenstra DL, Ramsey SD: Economic analyses of human genetics services: a systematic review. Genet Med 2005; 7: 519-523.

9 Rogowski W: Current impact of gene technology on healthcare. A map of economic assessments. Health Policy 2007; 80: 340-357.

10 Mardis ER: The $\$ 1000$ genome, the $\$ 100000$ analysis? Genome Med 2010; 2: 84.

11 Adair A, Hyde-Lay R, Einsiedel E, Caulfield T: Technology assessment and resource allocation for predictive genetic testing: a study of the perspectives of Canadian genetic health care providers. BMC Med Ethics 2009; 10: 6.

12 Rogowski WH, Hartz SC, John JH: Clearing up the hazy road from bench to bedside: a framework for integrating the fourth hurdle into translational medicine. BMC Health Serv Res 2008; 8: 194

13 Rogowski WH, Grosse SD, Khoury MJ: Challenges of translating genetic tests into clinical and public health practice. Nat Rev Genet 2009; 10: 489-495.

14 Rogowski WH, Grosse SD, Schmidtke J, Marckmann G: Criteria for fairly allocating scarce health-care resources to genetic tests: which matter most? Eur J Hum Genet 2014; 22: 25-31.

15 Severin F, Schmidtke J, Muhlbacher A, Rogowski WH: Eliciting preferences for priority setting in genetic testing: a pilot study comparing best-worst scaling and discretechoice experiments. Eur J Hum Genet 2013; 21: 1202-1208.

16 Daniels N: Just health: meeting health needs fairly. Cambridge [u.a.]: Cambridge University Press, 2008.

17 Daniels N, Sabin J: The ethics of accountability in managed care reform. Health Aff (Millwood) 1998; 17: 50-64.

18 Severin F, Hess W, Schmidtke J, Muhlbacher A, Rogowski W: Value judgments for priority setting criteria in genetic testing: a discrete choice experiment. Health Policy 2014.

19 Becker F, van EI CG, Ibarreta D et al: Genetic testing and common disorders in a public health framework: how to assess relevance and possibilities. Background document to the ESHG recommendations on genetic testing and common disorders. Eur J Hum Genet 2011; 19(Suppl 1): S6-S44.

20 McAllister M, Payne K, Macleod R, Nicholls S, Dian D, Davies L: Patient empowerment in clinical genetics services. J Health Psychol 2008; 13: 895-905.

21 Burn J, Gerdes AM, Macrae F et al: Long-term effect of aspirin on cancer risk in carriers of hereditary colorectal cancer: an analysis from the CAPP2 randomised controlled trial. Lancet 2011; 378: 2081-2087.

22 Palomaki GE, McClain MR, Melillo S, Hampel HL, Thibodeau SN: EGAPP supplementary evidence review: DNA testing strategies aimed at reducing morbidity and mortality from Lynch syndrome. Genet Med 2009; 11: 42-65.

23 Adank MA, Verhoef S, Oldenburg RA et al: Excess breast cancer risk in first degree relatives of CHEK2 ${ }^{*} 1100$ delC positive familial breast cancer cases. Eur J Cancer 2013; 49: 1993-1999.

24 Cholongitas E, Germani G, Burroughs AK: Prioritization for liver transplantation. Nature Rev Gastroenterol Hepatol 2010; 7: 659-668.

25 Nord E, Daniels N, Kamlet M: QALYs: some challenges. Value Health 2009; 12(Suppl 1): S10-S15.

26 Hatz MH, Schremser K, Rogowski WH: Is individualized medicine more cost-effective? A systematic review. Pharmacoeconomics 2014; 32: 443-455.

27 Stolk EA, Pickee SJ, Ament AH, Busschbach JJ: Equity in health care prioritisation: an empirical inquiry into social value. Health Policy 2005; 74: 343-355.

28 Fischer KE, Stollenwerk B, Rogowski WH: Link between process and appraisal in coverage decisions: an analysis with structural equation modeling. Med Decis Making 2013; 33: 1009-1025.

This work is licensed under a Creative Commons ported License. The images or other third party material in this article are included in the article's Creative Commons license, unless indicated otherwise in the credit line; if the material is not included under the Creative Commons license, users will need to obtain permission from the license holder to reproduce the material. To view a copy of this license, visit http://creative commons.org/licenses/by-nc-sa/3.0/

Supplementary Information accompanies this paper on European Journal of Human Genetics website (http://www.nature.com/ejhg) 